\title{
Enfoque Ontosemiótico de los Conocimientos y Competencias del Profesor de Matemáticas"
}

\author{
Onto-Semiotic Approach to Mathematics Teacher's \\ Knowledge and Competences
}

\author{
Juan D. Godino** \\ Belén Giacomone ${ }^{* * *}$ \\ Carmen Batanero ${ }^{* * * *}$ \\ Vicenç Font ${ }^{* * * * *}$
}

\begin{abstract}
Resumen
Diversos marcos teóricos proponen sistemas de categorías del conocimiento matemático para la enseñanza que ayudan a describir la práctica docente y a elaborar planes de formación de profesores. En este artículo se describe uno de estos sistemas, que incluye tanto los conocimientos como las competencias del profesor de matemáticas y está basado en el Enfoque ontosemiótico del conocimiento y la instrucción matemáticos. Las nociones de sistema de prácticas, configuración ontosemiótica, configuración didáctica, dimensión normativa e idoneidad didáctica, introducidas en dicho sistema teórico, son consideradas como herramientas de análisis de las prácticas matemáticas y didácticas. Se utilizan estas nociones como base para delimitar sub-competencias de la competencia general de análisis e intervención didáctica, propia del profesor de matemáticas. Las investigaciones que se vienen realizando usando este modelo teórico de articulación de los conocimientos y las competencias, apoyan la pertinencia y posibilidad de que el profesor conozca, comprenda y esté capacitado para aplicar las herramientas de análisis propuestas en su propia práctica profesional. Se incluye también la descripción sucinta de acciones formativas para lograr el desarrollo de los conocimientos didáctico-matemáticos y la competencia de análisis e intervención didáctica de los profesores de matemáticas.
\end{abstract}

\footnotetext{
* Versión revisada y ampliada de la comunicación presentada en el XX Simposio de la SEIEM con el título: Articulando conocimientos y competencias del profesor de matemáticas: el modelo CCDM.

Reconocimiento: Trabajo realizado en el marco de los proyectos de investigación EDU2012-31869, EDU201341141-P y EDU2015-64646-P, Ministerio de Economía y Competitividad (MINECO).

** Doctor por la Universidad de Granada (UGR). Profesor catedrático en la Universidad de Granada (UGR), Granada, España. Dirección postal: Departamento de Didáctica de la Matemática, Facultad de Ciencias de la Educación, Campus de Cartuja, s/n. 18071. Granada, España. E-mail: jgodino@ugr.es.

*** Maestría por la Universidad de Granada (UGR). Doctoranda en la Universidad de Granada (UGR), Granada, España. Dirección postal: Campus de Cartuja, s/n. 18071, Departamento de Didáctica de la Matemática, Facultad de Ciencias de la Educación. Granada, España. E-mail: giacomone @ correo.ugr.es.

**** Doctora por la Universidad de Granada (UGR). Profesora catedrática en la Universidad de Granada (UGR), Granada, España. Dirección postal: Departamento de Didáctica de la Matemática, Facultad de Ciencias de la Educación, Campus de Cartuja, s/n. 18071. Granada, España. E-mail: batanero@ugr.es.

${ }^{* * * * *}$ Doctor por la Universidad de Barcelona (UB). Profesor Titular en la Universidad de Barcelona (UB), Barcelona, España. Dirección postal: Departament de Didàctica de les Ciències Experimentals i la Matemàtica, Facultat de Formació del Professorat, Passeig de la Vall d'Hebrón, 171, 08035. Barcelona, España. E-mail: vfont@ub.edu.
} 
Palabras clave: Formación de Profesores. Enfoque Ontosemiótico. Conocimientos Didáctico-Matemáticos. Competencia de Análisis e Intervención Didáctica.

\begin{abstract}
Several theoretical frameworks propose different systems of mathematical knowledge for teaching that help describe teaching practices and develop plans for teacher training. This article describes one of these systems, which includes both knowledge and the competencies of a mathematics teacher, and it is based on the ontosemiotic approach of mathematical knowledge and instruction. The notions of this practices' system, ontosemiotic configuration, didactical configuration, normative dimension, and didactical suitability, are introduced in this theoretical system as analytical tools of mathematical and didactical practices. These tools are used as a basis to define five sub-competencies within the mathematics' teacher general competence of didactic analysis and intervention. Investigations are being carried out with this theoretical framework that articulates knowledge and competencies, and support the relevance and possibility that the teacher knows, understands and is able to apply the tools of analysis. Finally, it also includes a concise description on training actions for developing the didactical-mathematical knowledge, and the competence of analysis and didactical intervention for mathematics teachers.
\end{abstract}

Keywords: Teacher Training. Onto-Semiotic Approach. Didactic-Mathematical Knowledge. Didactical Competences.

\title{
1 Introducción
}

En las últimas décadas ha habido un incremento notable de las investigaciones sobre la formación de profesores de matemáticas, que se refleja en las revisiones incluidas en los handbooks de investigación en educación matemática (PONTE; CHAPMAN, 2006; SOWDER, 2007), la publicación de handbooks específicos (JAWORSKI; WOOD, 2008; LO; LEATHAM; ZOEST, 2014), y la publicación de revistas específicas como Journal of Mathematics Teacher Education, o en la serie de Springer Mathematics Teacher Education, con varios títulos publicados.

Se reconoce que la formación didáctica de los profesores es un campo de investigación científica y tecnológica que reclama atención por parte de la Didáctica de la Matemática, pues el desarrollo del pensamiento y de las competencias matemáticas básicas de los alumnos depende, de manera esencial, de dicha formación.

Hay un acuerdo generalizado en el área de educación matemática de que el profesor de matemáticas debe tener un cierto nivel de competencia matemática, es decir, ha de conocer y ser capaz de realizar las prácticas matemáticas necesarias para resolver los problemas matemáticos usualmente abordables por los estudiantes del nivel correspondiente, y debe saber articularlos con los bloques temáticos posteriores. Hay, también, un acuerdo generalizado de que el profesor debe tener un conocimiento especializado del propio contenido, de las transformaciones que se deben aplicar al mismo en los procesos de 
enseñanza y aprendizaje, así como de las interacciones del contenido matemático a enseñar con diversos factores (psicológicos, sociológicos, pedagógicos, tecnológicos etc.) que condicionan dichos procesos. La caracterización del conocimiento especializado del contenido matemático para la enseñanza ha sido abordada por diversos autores, aplicando marcos teóricos diversos (BALL; LUBIENSKI; MEWBORN, 2001; HILL; BALL; SCHILLING, 2008; ROWLAND; HUCKSTEP; THWAITE, 2005).

Schoenfeld y Kilpatrick (2008, p. 350) introducen la noción de proficiencia $^{l}$ en la enseñanza de las matemáticas, que puede ser interpretada como una referencia a los conocimientos (y competencias) que deberían tener los profesores para que su enseñanza se pueda considerar de calidad: "Una teoría de la proficiencia (en la enseñanza) dice lo que es importante - qué destrezas necesitan desarrollar las personas para llegar a ser proficientes". Se trata de extender la noción de proficiencia en la matemática escolar (introducida en KILPATRICK; SWAFFORD; FINDELL, 2001) donde se incluye: comprensión conceptual, fluencia procedimental, competencia estratégica, razonamiento adaptativo y disposición productiva.

Desde el punto de vista del sistema teórico Enfoque Ontosemiótico del conocimiento y la instrucción matemáticos (EOS) (GODINO; BATANERO; FONT, 2007) el profesor debe ser capaz de analizar la actividad matemática al resolver los problemas, identificando las prácticas, objetos y procesos puestos en juego, y las variables que intervienen en los enunciados, a fin de formular nuevos problemas y adaptarlos a cada circunstancia educativa. El desarrollo de dicha competencia es un desafío para los formadores de profesores por la diversidad de dimensiones y componentes a tener en cuenta. Una de ellas es el análisis de los propios conocimientos matemáticos, e implica adoptar una visión amplia que reconozca el papel central de la resolución de problemas en la generación del conocimiento.

En trabajos previos, realizados en el marco del EOS, se ha propuesto un modelo de categorías de los conocimientos didácticos-matemáticos del profesor de matemáticas (GODINO, 2009; PINO-FAN; GODINO, 2015) y también se ha abordado la descripción de las competencias profesionales del profesor de matemáticas, ligándolas básicamente con la competencia de describir, explicar y valorar los procesos de estudio matemático, o

\footnotetext{
${ }^{1}$ La palabra proficiencia no está incluida en el Diccionario de la Lengua Española. Existe el adjetivo proficiente, "Dicho de una persona: Que va aprovechando en algo". En español se podría usar el término eficacia: "Capacidad de lograr el efecto que se desea o se espera"; o también, eficiencia: "Capacidad de disponer de alguien o de algo para conseguir un efecto determinado", en nuestro caso, una enseñanza de la matemática de alta calidad.
} 
competencia de análisis didáctico (GODINO et al., 2012; RUBIO, 2012). En este trabajo presentamos un modelo que trata de articular las categorías de conocimientos y las competencias didácticas del profesor de matemáticas, usando las facetas y componentes de un proceso de estudio matemático descritas en el EOS (GODINO, 2013). Se toma como indicador de competencia "una acción eficaz realizada en un determinado contexto con una determinada finalidad" (FONT, 2011, p. 18). Por otra parte, las herramientas de análisis del EOS de sistema de prácticas, configuración ontosemiótica, configuración didáctica, dimensión normativa e idoneidad didáctica aportan criterios para definir sub-competencias de la competencia general de análisis didáctico.

A continuación, en la sección 2 se sintetizan las nociones básicas del EOS que sirven de fundamento para el modelo de Conocimientos y Competencias Didáctico-Matemáticas (modelo CCDM) del profesor de matemáticas. En la sección 3 se presentan las categorías de conocimientos matemáticos y didáctico-matemáticos a partir de las facetas y componentes que caracterizan los procesos de enseñanza y aprendizaje. En la sección 4 se describe la competencia de análisis e intervención didáctica y las cinco sub-competencias asociadas, derivadas de las herramientas de análisis que propone el EOS. En la sección 5 se muestran ejemplos de procesos formativos prototípicos, los cuales se vienen experimentando en diversas investigaciones para desarrollar las competencias y conocimientos específicos del profesor de matemáticas. El artículo concluye con algunas reflexiones finales.

\section{Fundamentos teóricos}

Como se ha indicado, partimos del Enfoque Ontosemiótico (EOS), sistema teórico que trata de integrar diversas aproximaciones y modelos teóricos usados en la investigación en Educación Matemática. Dicho enfoque se apoya en presupuestos antropológicos y semióticos sobre las matemáticas, y adopta principios didácticos de tipo socio-constructivista e interaccionista para el estudio de los procesos de enseñanza y aprendizaje.

El conjunto de nociones teóricas que, actualmente, componen el EOS, se clasifican en cinco grupos cada uno de los cuales permite analizar aspectos complementarios de los procesos de enseñanza y aprendizaje de las matemáticas (GODINO; BATANERO; FONT, 2007): 
(1) Sistema de prácticas (operativas y discursivas). El EOS adopta como elemento central la actividad de resolución de problemas en la construcción del conocimiento matemático (GODINO; BATANERO, 1994). La noción de sistema de prácticas (institucionales y personales) aporta la visión antropológica y pragmatista de las matemáticas e introduce las nociones de significado institucional y personal de los objetos matemáticos, distinguiendo diversos tipos de los mismos. La noción de significado institucional de referencia de un objeto o tema de estudio orienta el análisis sistemático de la literatura hacia la identificación de los diversos significados contextuales de los objetos y su articulación en un significado global u holístico. Este significado global se considera como la población de referencia (de situaciones-problemas) de la cual se seleccionarán muestras adecuadas a las circunstancias particulares de los procesos que se pretenden diseñar.

(2) Configuración de objetos y procesos matemáticos, emergentes e intervinientes en las prácticas matemáticas. La adopción de una noción interaccionista de objeto y pragmatista del significado (contenido de funciones semióticas) articula, de manera coherente, la concepción antropológica (Wittgenstein) con posiciones realistas (no platónicas) de las matemáticas. Los diversos medios de expresión (lenguajes) desempeñan el doble papel de instrumentos del trabajo matemático y de representación de los restantes objetos matemáticos. La noción de configuración ontosemiótica (de prácticas, objetos y procesos) responde a la necesidad de identificar los objetos y procesos implicados en las prácticas matemáticas que se realizan para la resolución de las situaciones-problemas cuya resolución competente se trata de desarrollar en los estudiantes (GODINO et al., 2011). El reconocimiento explícito de tales objetos y procesos permite prever conflictos potenciales y efectivos de aprendizaje, evaluar las competencias matemáticas de los estudiantes e identificar objetos (conceptos, proposiciones, procedimientos, argumentos) que deben ser recordados e institucionalizados en los momentos oportunos de los procesos de estudio.

(3) Configuración didáctica, entendida como sistema articulado de roles docentes y discentes, a propósito de una configuración de objetos y procesos matemáticos ligados a una situación-problema. Constituye la principal herramienta para el análisis de la instrucción matemática (CONTRERAS; GARCÍA; FONT, 2014; GODINO; CONTRERAS; FONT, 2006). Las configuraciones didácticas y su secuencia en trayectorias didácticas tienen en cuenta las facetas epistémica (conocimientos institucionales), cognitiva (conocimientos 
personales), afectiva, mediacional (recursos tecnológicos y temporales), interaccional y ecológica que caracterizan los procesos de estudio matemático.

(4) La dimensión normativa, o sistema de reglas, hábitos, normas que restringen y soportan las prácticas matemáticas y didácticas (GODINO et al., 2009), que generaliza las nociones de contrato didáctico (BROUSSEAU, 1990) y normas socio-matemáticas. El reconocimiento del efecto de las normas y meta-normas que intervienen en las diversas facetas que caracterizan los procesos de estudio matemático es uno de los factores explicativo de los fenómenos didácticos.

(5) La noción de idoneidad didáctica, como criterio general, relativo a las circunstancias contextuales, de adecuación y pertinencia de las acciones de los agentes educativos, de los conocimientos puestos en juego y de los recursos usados en un proceso de estudio matemático (GODINO, 2013). El sistema de indicadores empíricos identificados en cada una de las facetas constituye una guía para el análisis y reflexión sistemática que aporta criterios para la mejora progresiva de los procesos de enseñanza y aprendizaje.

En el marco del EOS las nociones de conocimiento y competencia se relacionan, teniendo en cuenta las conexiones entre práctica y objeto. La práctica, como acción orientada al fin de resolver un problema o realizar una tarea, conlleva una capacidad o competencia por parte del sujeto que la realiza. Pero la realización competente de una práctica implica la intervención de objetos interconectados que regulan y emergen de la misma, los cuales constituyen el conocimiento declarativo o discursivo correspondiente. La dialéctica entre práctica y objeto, entre competencia y conocimiento, se puede mostrar mediante el análisis ontosemiótico de las prácticas matemáticas puestas en juego para la resolución de un problema matemático.

\section{Modelo de conocimientos didáctico-matemáticos del profesor}

El eje central del EOS es la modelización del conocimiento matemático, en su doble faceta epistémica (institucional) y cognitiva (personal), basada en una aproximación antropológica (la matemática como actividad humana) y ontosemiótica (en la que la noción de objeto y significado son centrales). Esta modelización aporta las categorías primarias del conocimiento didáctico-matemático (GODINO, 2009; PINO-FAN; GODINO, 2015). 
Como se ha indicado en la introducción, el profesor de matemáticas tiene que conocer las matemáticas escolares del nivel educativo donde imparte, pero también debe poder articular esos conocimientos con los correspondientes a algunos niveles posteriores. Estos conocimientos constituyen el "conocimiento del contenido matemático per-se" (SCHEINER, 2015, p. 3250), que en el modelo propuesto desde el EOS constituyen, los conocimientos común (correspondiente al nivel en que se enseña) y ampliado (relativos a niveles superiores).

De acuerdo con la literatura citada, los conocimientos puramente matemáticos no son suficientes para que el profesor organice, implemente y evalúe los procesos de enseñanza y aprendizaje. Los factores que influyen en dichos procesos son complejos, y es necesario tener, también, un conocimiento más profundo de la matemática y su enseñanza, diferente del que adquieren los estudiantes, que llamaremos conocimiento didáctico-matemático.

En la Figura 1 se presenta el modelo de conocimiento didáctico-matemático, que se superpone al conocimiento matemático per-se (común y ampliado):

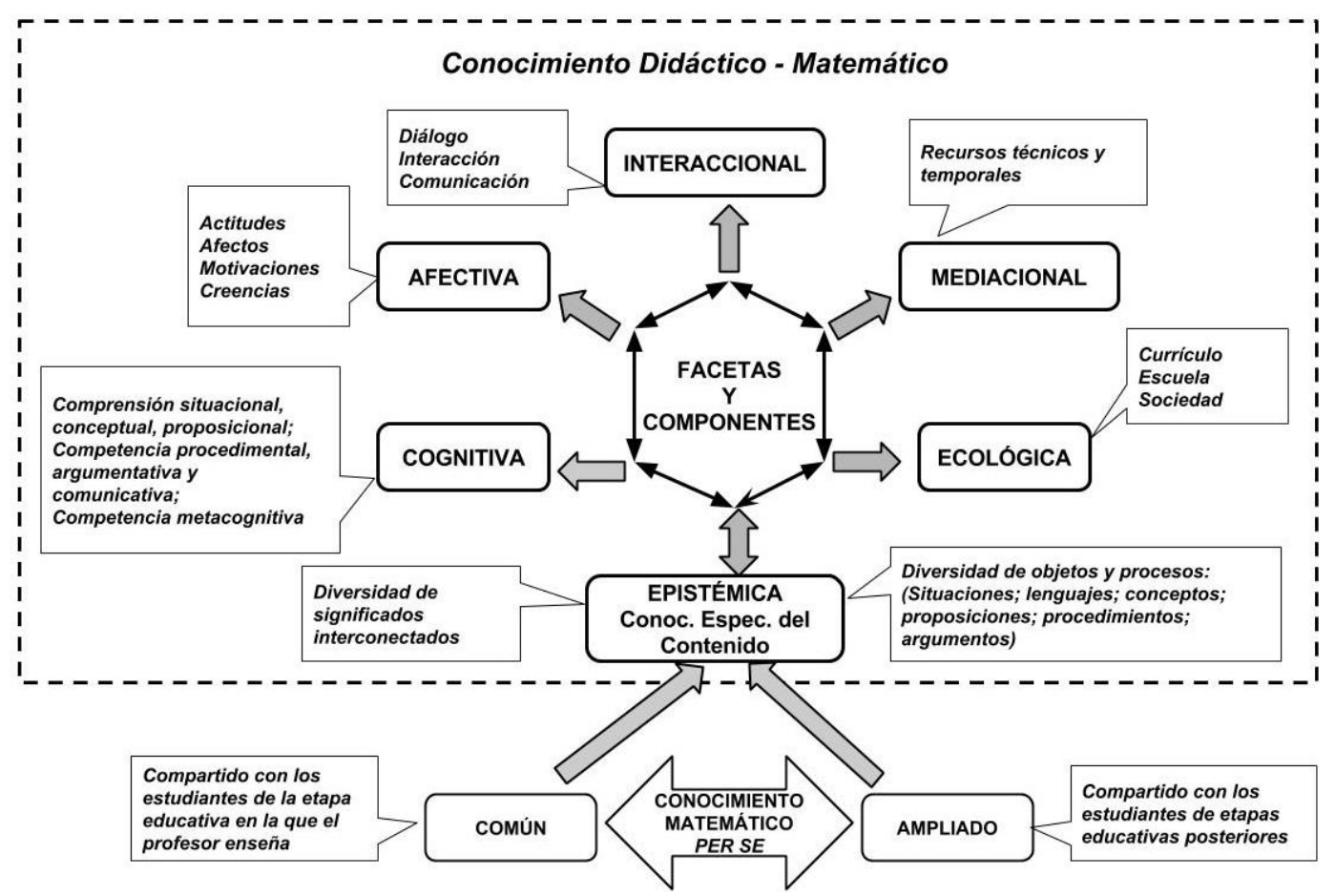

Figura 1 - Facetas y componentes del conocimiento del profesor

Fuente: Godino, Batanero, Font y Giacomone (2016, p. 292)

El modelo incluye las siguientes facetas:

- Faceta epistémica: es el conocimiento didáctico-matemático sobre el propio contenido, es decir, la forma particular en que el profesor de matemática comprende y conoce las matemáticas. Sería equivalente a lo que Ball, Lubienski y Mewborn (2001) 
denominan conocimiento especializado del contenido matemático, aunque en nuestro caso el EOS aporta un desglose analítico de sus elementos constituyentes.

- Faceta cognitiva: implica el conocimiento de cómo lo estudiantes aprenden, razonan y entienden las matemáticas y como progresan en su aprendizaje.

- Faceta afectiva: incluye los conocimientos sobre los aspectos afectivos, emocionales, actitudinales y creencias de los estudiantes con relación a los objetos matemáticos y al proceso de estudio seguido.

- Faceta interaccional: refiere al conocimiento sobre la enseñanza de las matemáticas, organización de las tareas, resolución de dificultades de los estudiantes, e interacciones que se puede establecer en el aula.

- $\quad$ Faceta mediacional: es el conocimiento de los recursos (tecnológicos, materiales y temporales) apropiados para potenciar el aprendizaje de los estudiantes.

- Faceta ecológica: implica las relaciones del contenido matemático con otras disciplinas, y los factores curriculares, socio-profesionales, políticos, económicos que condicionan los procesos de instrucción matemática.

Todas estas facetas forman parte del conocimiento especializado del profesor de matemáticas en la medida en que tales procesos ponen en juego algún contenido matemático, sea común o ampliado. Además, todas ellas se relacionan entre sí; por ejemplo, dada una tarea matemática determinada, el profesor debe ser capaz de movilizar la diversidad de significados que se ponen en juego (faceta epistémica) y también debe poder resolver la tarea, utilizando distintos procedimientos, mostrar diversas justificaciones y explicaciones, o bien variarla para adaptarla a los conocimientos de los alumnos (facetas instruccional y cognitiva).

Las categorías genéricas del conocimiento matemático para la enseñanza propuestas por otros modelos (p. e. MKT: HILL; BALL; SCHILLING, 2008) son desarrolladas en el modelo CCDM usando los componentes e indicadores de idoneidad didáctica del EOS (GODINO, 2013), según se muestra en la Figura 1. Por ejemplo, para el "conocimiento especializado del contenido" (SCK: HILL; BALL; SCHILLING, 2008, p. 377), que se corresponde aquí con la faceta epistémica del conocimiento didáctico-matemático, se propone tener en cuenta la diversidad de significados parciales de los objetos matemáticos y su interconexión. Pero además, la descripción de tales significados implica el reconocimiento de las configuraciones ontosemióticas correspondientes. 
El modelo de los conocimientos del profesor de matemáticas esquematizado en la Figura 1, proporciona otro modelo sobre los conocimientos del formador de profesores de matemáticas. Si lo aplicamos al profesor, se supone que los conocimientos se refieren a un proceso de estudio matemático en el que el profesor está implicado, y por tanto, la faceta cognitiva y afectiva se refieren a estudiantes de matemáticas. En el caso del formador de profesores, se trata de un proceso de estudio de didáctica de la matemática, donde los estudiantes son profesores de matemáticas en formación, a los cuáles se refieren las facetas afectiva y cognitiva. En la primera de dichas facetas se tienen en cuenta las creencias, y en la segunda, los procesos meta-cognitivos del profesor de matemáticas, las cuales deben ser conocidas y tenidas en cuenta por el formador. La formación de los profesores debe tener, también, en cuenta el conocimiento matemático per-se, ya que los conocimientos didácticos involucran, así mismo, al contenido matemático.

A continuación, articulamos la noción de competencia con la de conocimiento didáctico-matemático y proponemos un desglose operativo de la competencia general de análisis e intervención didáctica utilizando las herramientas teóricas escritas en la sección 2.

\section{Competencias didácticas específicas}

Se espera que el profesor de matemáticas esté capacitado para abordar los problemas didácticos básicos que están presentes en la enseñanza. Además, en las prácticas didácticas puestas en juego en la resolución de problemas didácticos también intervienen objetos matemáticos y didácticos específicos (conocimientos), que deben ser conocidos por el profesor.

Para desarrollar estas competencias y conocimientos, en el EOS se aportan determinadas herramientas teóricas y metodológicas, dando lugar a una competencia general de diseño e intervención didáctica, propia del profesor de matemáticas; dicha competencia general se compone de cinco sub-competencias, que se describen a continuación.

\subsection{Competencia de análisis significados globales}

Esta competencia se requiere cuando el profesor trata de dar respuesta a las cuestiones: 
- ¿ ¿Cuáles son los significados de los objetos matemáticos implicados en el estudio del contenido pretendido?

- ¿Cómo se articulan entre sí?

En la fase preliminar del proceso de diseño instruccional, los significados son entendidos, de manera pragmática, como sistemas de prácticas cuyo objetivo es construir un modelo de referencia que delimite los tipos de problemas abordados y las prácticas operativas y discursivas requeridas para su resolución.

Supongamos que se estudian las fracciones. El profesor debe poder caracterizar tanto las prácticas institucionales (diferentes significados institucionales de las fracciones; por ejemplo, como razón, proporción, parte-todo etc.), teniendo en cuenta los diversos contextos de usos donde tales problemas se presentan, como también las prácticas personales esperadas del alumno (significados personales que puedan adquirir los alumnos sobre las fracciones).

El conocimiento de la noción "sistemas de prácticas matemáticas operativas y discursivas, y sus diversos tipos" (GODINO; BATANERO; FONT, 2007, p. 129) se corresponde con una competencia de análisis de significados globales. El foco de atención, ahora, es la identificación de las situaciones-problemas que aportan los significados parciales o sentidos para los objetos, o temas matemáticos bajo estudio, y las prácticas operativas y discursivas que se deben poner en juego en su resolución. En el ejemplo dado, la búsqueda de situaciones que de sentido a los diferentes significados de las fracciones.

\subsection{Competencia de análisis ontosemiótico de prácticas matemáticas}

En la resolución de problemas o tareas matemáticas interviene y emerge una trama de objetos que hacen posible la realización de las prácticas correspondientes. Dichos objetos deben ser reconocidos, de manera explícita, por el alumno para progresar en la construcción del conocimiento. La identificación por parte del profesor de los objetos y procesos intervinientes en las prácticas matemáticas es una competencia que le permitirá comprender la progresión de los aprendizajes, gestionar los necesarios procesos de institucionalización y evaluar las competencias matemáticas de los alumnos. Se trata, por tanto, de responder a las cuestiones: 
- ¿Cuáles son las configuraciones de objetos y procesos matemáticos implicados en las prácticas que constituyen los diversos significados de los contenidos pretendidos? (configuraciones epistémicas).

- ¿Cuáles son las configuraciones de objetos y procesos puestas en juego por los alumnos en la resolución de los problemas? (configuraciones cognitivas).

El profesor de matemáticas debe conocer y comprender la idea de configuración de objetos y procesos y ser capaz de usarla de manera competente en los procesos de diseño didáctico. Se trata de la competencia de análisis ontosemiótico de las prácticas matemáticas implicadas en la solución de las tareas instruccionales.

\subsection{Competencia de análisis y gestión de configuraciones didácticas}

El profesor de matemáticas debe conocer y comprender la noción de configuración didáctica (GODINO; CONTRERAS; FONT, 2006), introducida como una herramienta para el análisis de las interacciones, personales y materiales, en los procesos de estudio matemático. Es decir, debe conocer los diversos tipos de configuraciones didácticas que se pueden implementar y sus efectos sobre el aprendizaje de los estudiantes. Además, ha de tener competencia para su uso pertinente en la implementación de los diseños instruccionales, la cual se puede describir como competencia de gestión de configuraciones didácticas. Debe poder responder al problema docente de cómo enseñar un contenido específico, que en el marco del EOS se concreta del siguiente modo:

- $\quad$ ¿Qué tipos de interacciones entre personas y recursos se implementan en los procesos instruccionales y cuáles son sus consecuencias sobre el aprendizaje?

- ¿Cómo gestionar las interacciones para optimizar el aprendizaje?

\subsection{Competencia de análisis normativo}

Las distintas fases del proceso de diseño didáctico están apoyadas y son dependientes de una trama compleja de normas, de distinto origen y naturaleza (GODINO et al., 2009) y meta-normas (D’AMORE; FONT; GODINO, 2007), cuyo reconocimiento explícito es necesario para poder comprender el desarrollo de los procesos de estudio matemático y encauzarlos hacia niveles óptimos de idoneidad. Por ejemplo, al estudiar las fracciones 
aparecen normas sobre su escritura o su forma de representación gráfica. También, hay normas no matemáticas, como el tiempo dedicado al tema de las fracciones, libro que tiene el alumno o fechas en que se realiza la evaluación.

El profesor de matemáticas debe conocer, comprender y valorar la dimensión normativa y usarla de manera competente, siendo necesario, por tanto, diseñar acciones formativas para un uso instrumental de la misma. Se trata, ahora, de desarrollar la competencia de análisis normativo de los procesos de estudio matemático para responder a las cuestiones:

- ¿ ¿Qué normas condicionan el desarrollo de los procesos instruccionales?

- ¿ ¿Quién, cómo y cuándo se establecen las normas?

- ¿ ¿Cuáles y cómo se pueden cambiar para optimizar el aprendizaje matemático?

\subsection{Competencia de análisis y valoración de la idoneidad didáctica}

Las cuestiones profesionales, mencionadas anteriormente, implican una mirada a nivel microscópico de la práctica docente, esto es, realizar análisis pormenorizados de actividades de resolución de problemas o de actividades de enseñanza y aprendizaje puntuales. En el marco del EOS se ha introducido la noción de idoneidad didáctica, que orienta el análisis a nivel macroscópico de los procesos de estudio matemático. La idoneidad didáctica de un proceso de instrucción se define como el grado en que dicho proceso (o una parte del mismo) reúne ciertas características que permiten calificarlo como idóneo (óptimo o adecuado) para conseguir la adaptación entre los significados personales logrados por los estudiantes (aprendizaje) y los significados institucionales pretendidos o implementados (enseñanza), teniendo en cuenta las circunstancias y recursos disponibles (entorno).

Fijado un tema específico en un contexto educativo determinado, por ejemplo, el estudio de las ecuaciones de segundo grado en educación secundaria, la noción de idoneidad didáctica (GODINO, 2013) lleva a plantear las cuestiones,

- ¿Cuál es el grado de idoneidad didáctica del proceso de enseñanza-aprendizaje implementado sobre las ecuaciones de segundo grado?

- ¿Qué cambios se deberían introducir en el diseño e implementación del proceso de estudio para incrementar su idoneidad didáctica en un próximo ciclo de experimentación? 
Para poder emitir un juicio fundamentado sobre la idoneidad didáctica de un proceso de estudio matemático es imprescindible realizar una reconstrucción de los significados de referencia didáctica del tema correspondiente. Ello requiere proceder a una revisión sistemática de los resultados de las investigaciones e innovaciones realizadas en educación matemática sobre los aspectos epistémicos, ecológicos, cognitivos, afectivos, interaccionales y mediacionales. Esto lleva a plantear una cuestión previa:

- ¿Cuáles son los conocimientos didáctico-matemáticos resultados de las investigaciones e innovaciones previas realizadas sobre la enseñanza-aprendizaje de las ecuaciones de segundo grado?

La noción de idoneidad didáctica se ha introducido como una herramienta de apoyo para la reflexión global sobre la práctica didáctica, su valoración y mejora progresiva. El profesor de matemáticas debe conocer, comprender y valorar esta herramienta y adquirir competencia para su uso pertinente. Se trata de la competencia de análisis de la idoneidad didáctica de los procesos de estudio matemáticos.

\subsection{Competencia general de análisis e intervención didáctica y conocimientos didácticos}

Las competencias descritas anteriormente se pueden considerar como subcompetencias de una más amplia, propia del profesor de matemáticas, que es la de análisis e intervención didáctica, como se representa en la Figura 2.

La articulación de las competencias y conocimientos didácticos se puede hacer, de manera natural, en el marco del EOS. En efecto, las prácticas matemáticas y didácticas son entendidas como acciones del sujeto orientadas hacia el fin de resolver un problema o realizar una tarea (no son meras conductas o comportamientos). Estas prácticas pueden ser de tipo discursivo-declarativo, indicando la posesión de conocimientos, o de tipo operatorioprocedimental, indicando la posesión de una capacidad o competencia. Ambos tipos de prácticas están imbricados, de manera que la realización eficiente de prácticas operatorias conlleva la puesta en acción de conocimientos declarativos, los cuales se pueden referir a la descripción de los instrumentos usados o a resultados previamente obtenidos que deben ser activados. A su vez, la comprensión de los conocimientos declarativos requiere que el sujeto esté enfrentado a las situaciones que proporcionan la razón de ser de tales conocimientos e implicado (disposición para la acción) en su resolución eficiente (Figura 2). 


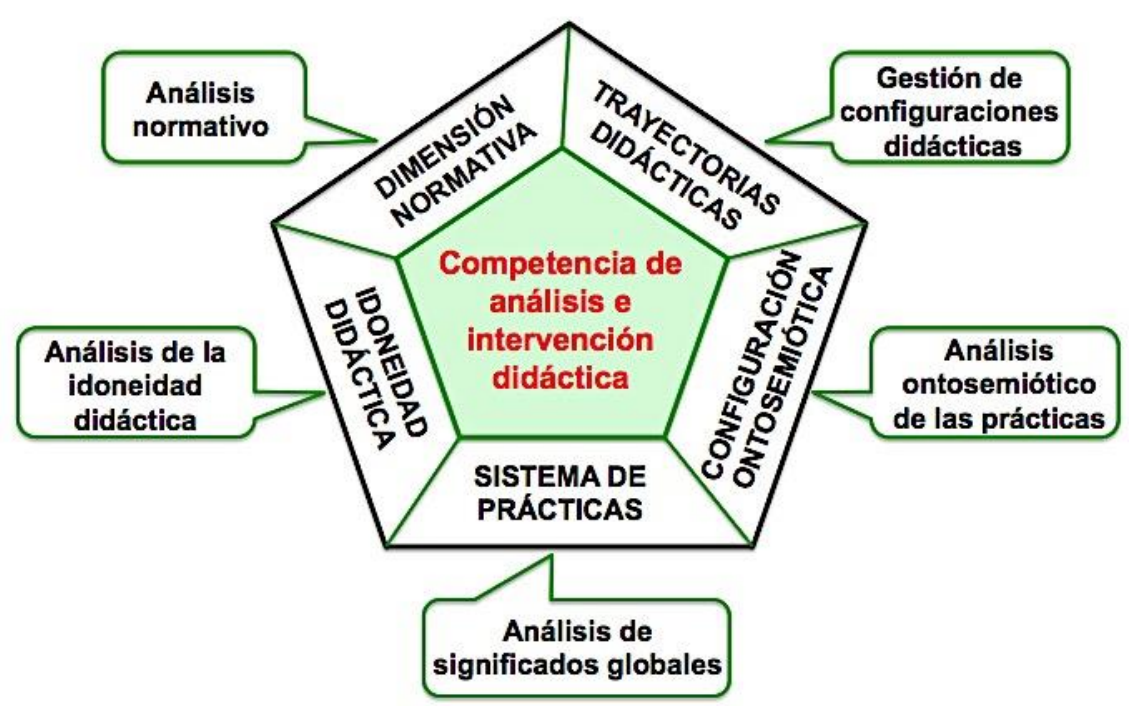

Figura 2 - Componentes de la competencia de análisis e intervención didáctica Fuente: Godino, Batanero, Font y Giacomone (2016, p. 295)

\section{Procesos formativos para desarrollar las competencias y conocimientos específicos del profesor de matemáticas}

Para la adquisición de los conocimientos y competencias didáctico-matemáticas descritos en los apartados anteriores, es necesario diseñar e implementar procesos formativos que permitan contribuir al aprendizaje de los futuros profesores, a su crecimiento profesional y al cambio en las nuevas prácticas de instrucción para la educación matemática.

Con la intención de hacer operativos los mencionados aspectos teóricos, en este apartado describimos tres acciones formativas orientadas al logro de dichos conocimientos y competencias. Las mismas son ejemplos del tipo de experiencias que estamos desarrollando en el contexto de cursos de máster para la formación inicial de profesores de educación secundaria. La primera se centra en el desarrollo de la competencia de reconocimiento de objetos y procesos en la resolución de un problema sobre fracciones (análisis ontosemiótico de las prácticas matemáticas); la segunda en el análisis y valoración de una experiencia de estudio de la semejanza de triángulos en segundaria, a partir de la información parcial ofrecida por una videograbación. El tercer tipo de intervención formativa está relacionado con el análisis de la idoneidad didáctica de experiencias docentes vividas por los propios profesores en formación en la fase de prácticas de enseñanza. 
Cada una de las siguientes acciones representa una parte de una secuencia formativa más amplia, en las que se emplean varias sesiones de clase, acompañadas de momentos de trabajo individual y grupal, presencial y no presencial, discusiones grupales e institucionalizaciones por parte del docente. En un primer momento, se presenta el problema didáctico que se debe abordar, y se utiliza el diseño de una tarea para explorar los significados personales iniciales de los estudiantes en formación, respecto a la competencia que se quiere desarrollar. Seguidamente, se incorpora la lectura y discusión de un documento introductorio en el que se describe el instrumento propuesto a los futuros profesores, ejemplificando su uso en un caso y mostrando el tipo de resultados que se pueden obtener. Finalmente se implementan acciones, como las siguientes, en las que los futuros profesores aplican el instrumento a nuevos casos para adquirir, progresivamente, destreza en su aplicación eficiente.

Es importante tomar conciencia que lograr la comprensión y el dominio de las herramientas teóricas propuestas no es instantáneo, ni es posible lograrlo con lecciones o discursos declarativos aislados. Se requiere tiempo y un periodo de práctica guiada.

\subsection{Prácticas, objetos y procesos intervinientes en la resolución de un problema de fracciones}

En esta acción formativa se parte de una tarea matemática sobre la cual se plantean las siguientes consignas:

a) Resuelve la tarea.

b) Describe el procedimiento seguido en la resolución, indicando la secuencia de prácticas elementales que has realizado para resolver la tarea y añade las explicaciones necesarias que justifican las respuestas.

c) Completa la tabla incluida a continuación en la que se identifican los procesos de significación implicados (añade las filas necesarias)

\begin{tabular}{|l|l|l|}
\hline $\begin{array}{l}\text { Uso e intencionalidad de las } \\
\text { prácticas }\end{array}$ & $\begin{array}{l}\text { Enunciado y secuencia de } \\
\text { prácticas elementales para } \\
\text { resolver la tarea }\end{array}$ & $\begin{array}{l}\text { Objetos referidos en las prácticas } \\
\text { (conceptos, proposiciones, } \\
\text { procedimientos ....) }\end{array}$ \\
\hline$-\ldots$ & $-\quad \ldots$ & - \\
\hline
\end{tabular}


d) Identifica otros procesos matemáticos implicados en la solución (particularizacióngeneralización, materialización-idealización, representación-significación, ...) (ver GODINO; BATANERO; FONT, 2007, p. 132).

e) Destaca entre las prácticas, objetos y procesos identificados cuáles consideras potencialmente conflictivos para los estudiantes.

f) Enuncia variantes de la tarea e identifica los cambios que se producen en los conocimientos puestos en juego en cada variación.

Es importante aclarar que cuando los estudiantes en formación se enfrentan a este tipo de consignas, existe una instrucción previa, tal como se ha mencionado anteriormente, y por tanto, un lenguaje compartido. En este sentido, los participantes comprenden el significado de los elementos de la tabla de la consigna c) y de los tipos de procesos que se les pide identificar.

En la Figura 3 se propone un ejemplo de tarea sobre la cual se plantean las consignas indicadas. En este caso, se trata de un problema matemático sobre fracciones acompañado por una posible solución dada por un alumno. En esta acción, no se pretende que los futuros profesores resuelvan, sino que apliquen el análisis ontosemiótico a la solución dada, esto es, realizar un análisis de la respuesta del alumno. De esta manera, es posible comprender qué conocimientos efectivos moviliza un sujeto cuando resuelve el problema dado.

\begin{tabular}{|c|c|}
\hline $\begin{array}{l}\text { Tarea: Un estudiante resuelve el siguiente } \\
\text { problema según se indica en la figura adjunta. } \\
\text { ¿Es correcta la respuesta dada por el estudiante? } \\
\text { Justifica la respuesta } \\
\text { Problema (fracción de alcohol en un Martini): } \\
\text { Un Martini es un cóctel que se hace con } 5 \\
\text { partes de ginebra y l parte de vermut. } \\
\text { Supongamos que } 2 / 5 \text { de la ginebra es alcohol y } \\
\text { que 1/6 del vermut es alcohol. ¿Qué fracción de } \\
\text { alcohol lleva un Martini? Resuelve el problema } \\
\text { usando un diagrama de áreas. }\end{array}$ & 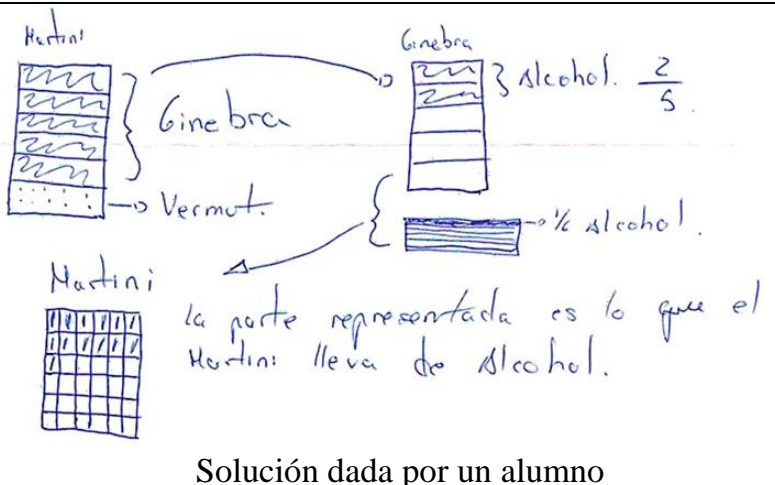 \\
\hline
\end{tabular}

Figura 3 - Tarea para realizar el análisis ontosemiótico Fuente: Giacomone y Godino (2016, p. 5)

En Giacomone y Godino (2016) se presenta el análisis a priori de esta tarea, mostrando las oportunidades que ofrece la herramienta de configuración ontosemiótica de prácticas, objetos y procesos para comprender las matemáticas que se ponen en juego en el desarrollo de la tarea y apoyar la puesta en común en clase. Además, en dicho trabajo, se describen los resultados obtenidos al implementar la tarea con un grupo de 52 estudiantes en 
formación, destacando la complejidad y la importancia que implica el diseño e implementación de este tipo de acciones para el desarrollo profesional docente.

\subsection{Análisis y valoración de una experiencia de estudio de la semejanza de triángulos en secundaria}

En este ejemplo, la acción formativa trata de desarrollar conjuntamente las competencias de describir, explicar y valorar experiencias de aula globales, poniendo en juego las herramientas configuración didáctica, dimensión normativa e idoneidad didáctica.

Describimos, a continuación, un ejemplo de acción formativa que fue diseñada y analizada a priori, implementada con un grupo de 27 futuros profesores y valorada posteriormente. Luego de impartir un proceso instruccional previo, se propone a los estudiantes las consignas mostradas en la Figura 4, para reflexionar sobre un proceso de enseñanza y aprendizaje. Se inicia con el visionado de un episodio de clase video-grabada sobre una clase de secundaria en la que un grupo de alumnos aprenden el cálculo de alturas inaccesibles, aplicando la semejanza de triángulos, con una metodología fijada por la Reforma en el sistema educativo mexicano.

Las consignas están divididas en tres partes claves. En la primera se trata de describir qué está sucediendo en el proceso de estudio observado. En la segunda, de explicar por qué sucede. A partir de la información recogida en esos dos apartados, los estudiantes pueden construir una base para emitir un juicio de valoración razonable y responder el tercer punto en base a qué aspectos se podrían mejorar.

En la siguiente dirección de YouTube encontramos un video (10 minutos de duración) de una clase de matemáticas: http://www.youtube.com/watch?v=60s_0Ya2-d8. Después de visionado el vídeo, y trabajando en equipos, elaborar un informe respondiendo a las siguientes cuestiones:

1) Descripción:

a) ¿Qué contenido matemático se estudia?

b) ¿Qué significados caracterizan el contenido estudiado?

c) ¿Cuál es el contexto y nivel educativo en que tiene lugar la clase?

d) ¿Qué hace el profesor?

e) ¿Qué hace el alumno?

f) ¿Qué recursos se utilizan?

g) ¿Qué conocimientos previos deben tener los alumnos para poder abordar la tarea?

h) ¿Qué dificultades/conflictos de aprendizaje se manifiestan?

i) ¿Qué normas (regulaciones, hábitos, costumbres) hacen posible y condicionan el desarrollo de la clase?

2) Explicación:

a) ¿Por qué se estudia ese contenido?

b) ¿Por qué se usa un problema realista para estudiar el contenido?

c) ¿Por qué actúa el docente de la manera en que lo hace? 
d) ¿Por qué actúa los alumnos de la manera en que lo hacen?

3) Valoración:

Emitir un juicio razonado sobre la enseñanza observada en las siguientes facetas, indicando algunos cambios que se podrían introducir para mejorarla:

a) Epistémica (contenido matemático estudiado)

b) Ecológica (relaciones con otros temas, currículo)

c) Cognitiva (conocimientos previos, aprendizaje, ...)

d) Afectiva (interés, motivación, ...)

e) Interaccional (modos de interacción entre profesor y estudiantes)

f) Mediacional (recursos usados)

4) Limitaciones de la información disponible:

¿Qué información adicional sería necesario tener para que el análisis realizado fuera más preciso y fundamentado?

Figura 4 - Análisis de un episodio de clase video-grabado

Fuente: Elaboración propia de esta investigación

Con la implementación de esta acción formativa, se pone en evidencia que el sistema de facetas, componentes e indicadores de idoneidad didáctica permite poner en acción puntos de decisión claves para la reflexión, identificando fortalezas, debilidades y mostrando la apertura hacia la introducción de cambios fundamentados. Asimismo, los participantes de este estudio han valorado positivamente este tipo de situaciones didácticas para su formación, destacándolas como necesarias, principalmente, para la planificación e implementación de sus propias clases.

\subsection{Valoración de la idoneidad didáctica de una experiencia de enseñanza vivida por el profesor}

Una acción formativa más completa que las descritas en los apartados anteriores se ha diseñado teniendo en cuenta la experiencia de los futuros profesores en la fase de prácticas de enseñanza. Ahora, se trata de que el profesor reflexione sobre el diseño, implementación y evaluación de una experiencia de enseñanza de un tema específico en un contexto educativo fijado. Un ejemplo es el trabajo de Posadas y Godino (2014), donde una estudiante de máster describe y analiza, aplicando la noción de idoneidad didáctica, el proceso de enseñanza de la ecuación cuadrática en $3^{\circ}$ de educación secundaria, que experimentó personalmente en siete sesiones de clase durante el periodo de prácticas de enseñanza.

La valoración de la idoneidad didáctica, y la consiguiente identificación de propuestas fundamentadas de cambio para el rediseño de la experiencia, requiere recopilar y sintetizar los conocimientos didáctico-matemáticos producidos en la investigación e innovación sobre la enseñanza y aprendizaje de las ecuaciones cuadráticas. La aplicación de los criterios de 
idoneidad didáctica ayudó a sistematizar los conocimientos didácticos y su aplicación a la reflexión y mejora progresiva de la práctica de la enseñanza.

Este tipo de acción formativa se viene experimentando ampliamente en los programas de maestría de diversas universidades españolas y latinoamericanas, como se puede ver en Breda, Font y Lima (2015).

Otro ejemplo más complejo se describe en el trabajo de Beltrán-Pellicer (2016), donde el objetivo es evaluar la idoneidad didáctica de la implementación de una unidad didáctica sobre azar y probabilidad para, luego, proponer modificaciones que puedan mejorar de forma justificada su idoneidad didáctica en ciclos sucesivos. Este trabajo resulta interesante, dado que para poder valorar las seis facetas (epistémica, ecológica, cognitiva, afectiva, instruccional y mediacional) que interactúan en el proceso de estudio, el autor considera necesario realizar, en primer lugar: una síntesis de los resultados de las innovaciones e investigaciones previas realizadas sobre la enseñanza-aprendizaje de dicho tema; y en segundo lugar: a partir de los componentes e indicadores generales planteados en Godino (2013), generar un sistema de componentes e indicadores específicos sobre la probabilidad y el azar. Esto pone de manifiesto, cómo el uso competente de la herramienta de idoneidad didáctica es útil tanto para el profesor, como para el formador e investigador en educación matemática.

\section{Reflexiones finales}

En este artículo, hemos ampliado el modelo de conocimientos didáctico-matemáticos (CDM) desarrollado en trabajos previos, incluyendo la noción de competencia didáctica del profesor de matemáticas (GODINO et al., 2016). Las facetas, componentes y herramientas teóricas que propone el EOS permiten mostrar la relación estrecha y cooperativa entre las nociones de conocimiento y competencia, y proporcionar criterios para categorizarlos. Como se desarrolla en Godino (2002), atribuimos un significado distinto y complementario a las nociones de competencia y comprensión matemática, relacionándolas con los componentes operatorios y discursivos del conocimiento, respectivamente. No obstante, otro uso más amplio que se suele hacer de competencia, sobre todo en los contextos de innovación curricular, es para referir a todo el complejo cognitivo-afectivo, que comprende tanto los 
aspectos operatorios como discursivos del conocimiento matemático, e incluso la disposición para la acción.

Aunque el planteamiento que hemos hecho en el artículo es básicamente teórico, mostrando la coherencia del modelo en base a los supuestos, dimensiones y herramientas del EOS, resaltamos que se vienen realizando numerosas investigaciones experimentales sobre los diversos componentes del modelo, como se puede ver en el apartado de formación de profesores de la web, enfoqueontosemiótico.ugr.es. Dado el carácter dinámico e inclusivo del EOS se puede apreciar, no obstante, una cierta evolución de las nociones y herramientas de análisis. Así, la noción de análisis de significados globales se corresponde con la reconstrucción de los significados de referencia institucionales de los objetos matemáticos realizada en trabajos previos; por ejemplo en Wilhelmi, Godino y Lacasta (2007) se realiza un análisis del significado holístico de la igualdad de números reales, y en Batanero y Díaz (2007) sobre los diversos significados de la probabilidad y la identificación de conflictos de significado.

Respecto de la técnica de análisis ontosemiótico, en Godino, Batanero y Roa (2005) se analizan las prácticas matemáticas en la resolución de problemas combinatorios, poniendo el acento en la identificación de los tipos de problemas, los lenguajes, conceptos, procedimientos, proposiciones y argumentos implicados, tanto en la solución por un sujeto epistémico como por estudiantes individuales. Aquí, se puede apreciar una cierta evolución de la técnica de análisis; se propone, ahora, realizar previamente la descomposición en prácticas elementales e identificar la configuración de objetos y procesos, en particular, el de significación, en cada una de las prácticas elementales.

La aplicación de las herramientas de análisis de significados globales y análisis ontosemiótico de las prácticas permite dar una respuesta al problema de la naturaleza del conocimiento especializado del contenido matemático. El conocimiento académico o profesional de un objeto o contenido matemático, por ejemplo, la probabilidad, se centra usualmente en el significado formal/axiomático del mismo, que es el usado en la solución de los problemas que se deben abordar en dicho contexto. El profesor de matemáticas debe conocer los diversos significados, tanto informales como formales, y sus interconexiones (aproximación intuitiva, frecuencial, clásica, lógica, subjetiva, axiomática) (BATANERO; DÍAZ, 2007). Pero incluso para cada significado parcial del objeto, y la resolución de las tareas prototípicas que los caracterizan, es necesario que el profesor conozca la trama de 
objetos y procesos implicados, con el fin de poder planificar la enseñanza, gestionar las interacciones en el aula, comprender las dificultades y evaluar los niveles de aprendizaje de los estudiantes.

En algunas orientaciones curriculares de matemáticas se está proponiendo que los estudiantes aprendan por competencias (MECD, 2015), o estándares de la práctica matemática (NGACBP, 2011), discriminando tipos de competencias matemáticas a desarrollar y niveles de complejidad de las tareas. Esto supone un reto para los profesores, tanto para evaluar como para desarrollar dichas competencias en los estudiantes. Como se muestra en la tesis doctoral de Rubio (2012), la realización de tales evaluaciones, de manera objetiva, requiere hacer los análisis pormenorizados de prácticas, objetos y procesos descritos anteriormente, aplicando la noción de configuración ontosemiótica.

El modelo CCDM abre, en consecuencia, un programa de investigación y desarrollo focalizado en el diseño, experimentación y evaluación de intervenciones formativas que promuevan el desarrollo profesional del profesor de matemáticas, teniendo en cuenta las distintas categorías de conocimientos y competencias didácticas descritas en este trabajo.

\section{Referencias}

BALL, D.; LUBIENSKI, S.; MEWBORN, D. Research on teaching mathematics: the unsolved problem of teachers' mathematical knowledge. In: RICHARDSON, V. (Ed.). Handbook of Research on Teaching. Washington: Ed. American Educational Research Association, 2001. p. 433-456.

BATANERO, C.; DÍAZ, C. The meaning and understanding of mathematics. In: FRANÇOIS, K.; BENDEGEM, J. P. (Ed.). Philosophical Dimensions in Mathematics Education. Berlín: Ed. Springer, 2007. p. 107-127.

\section{BELTRÁN-PELLICER, P. Evaluación de la Idoneidad Didáctica de una Experiencia de Enseñanza del Azar y Probabilidad en Tercer Curso ESO. 2016. 126 f. Tesis (Maestría en Educación) - Facultad de Ciencias de la Educación, Universidad de Granada, Granada, 2016. Disponible en: 〈http://www.ugr.es/ jgodino/Tesis_master/TFM_PBeltran.pdf $>$. Acceso en: 7, dic. 2016.}

BREDA, A.; FONT, V.; LIMA, V. M. R. A noção de idoneidade didática e seu uso na formação de professores de matemática. Jornal Internacional de Estudos em Educação Matemática, São Paulo, v. 8, n. 2, p. 1-41, 2015.

BROUSSEAU, G. Le contrat didactique: le milieu. Recherches en Didactique des Mathématiques, Grenoble, v. 9, n. 3, p. 309-336, 1990.

CONTRERAS, A; GARCÍA, M.; FONT, V. Análisis de un proceso de estudio sobre la enseñanza del límite de una función. Bolema, Rio Claro, v. 26, n. 42, p. 667-690, abr. 2014. 
D’AMORE, B.; FONT, V.; GODINO, J. D. La dimensión metadidáctica en los procesos de enseñanza y aprendizaje de la matemática. Paradigma, Maracay, v. 28, n. 2, p. 49-77, dic. 2007.

FONT, V. Competencias profesionales en la formación inicial de profesores de matemáticas de secundaria. Unión, San Cristóbal de La Laguna, n. 26, p. 9-25, jun. 2011.

GIACOMONE, B; GODINO, J. D. Experiencia formativa para desarrollar una competencia didácticomatemática de futuros profesores. In: XVI CONGRESO DE ENSEÑANZA Y APRENDIZAJE DE LAS MATEMÁTICAS. MATEMÁTICAS, NI MÁS NI MENOS, 16., 2016, Jerez. Actas...Jerez: CEAM, 2016. p. 1-10.

GODINO, J. D. Prospettiva semiotica della competenza e della comprensione matematica. La Matematica e la sua Didattica, Bolonia, v. 16, n. 4, p. 434-450, 2002.

GODINO J. D. Categorías de análisis de los conocimientos del profesor de matemáticas. Unión, San Cristóbal de La Laguna, n. 20, p. 13-31, dic. 2009.

GODINO J. D. Indicadores de idoneidad didáctica de procesos de enseñanza y aprendizaje de las matemáticas. Cuadernos de Investigación y Formación en Educación Matemática, San José, n. 11, p. 111-132, dic. 2013.

GODINO, J. D.; BATANERO, C. Significado institucional y personal de los objetos matemáticos. Recherches en Didactique des Mathématiques, Grenoble, v. 14, n. 3, p. 325-355, 1994.

GODINO, J. D.; BATANERO, C.; ROA, R. An onto-semiotic analysis of combinatorial problems and the solving processes by university students. Educational Studies in Mathematics, Dordrecht, v. 60, n. 1, p. 3-36, 2005.

GODINO, J. D.; CONTRERAS, A.; FONT, V. Análisis de procesos de instrucción basado en el enfoque ontológico-semiótico de la cognición matemática. Recherches en Didactiques des Mathematiques, Grenoble, v. 26, n. 1, p. 39-88, 2006.

GODINO, J. D.; BATANERO, C.; FONT, V. The onto-semiotic approach to research in mathematics education. ZDM Mathematics Education, Berlín, v. 39, n. 1, p. 127-135, enero. 2007.

GODINO, J. D.; FONT, V.; WILHELMI, M. R.; CASTRO, C. Aproximación a la dimensión normativa en didáctica de las matemáticas desde un enfoque ontosemiótico. Enseñanza de las Ciencias, Barcelona, v. 27, n. 1, p. 59-76, 2009.

GODINO, J. D.; FONT, V.; WILHELMI, M. R.; LURDUY, O. Why is the learning of elementary arithmetic concepts difficult? Semiotic tools for understanding the nature of mathematical objects. Educational Studies in Mathematics, Dordrecht, v. 77, n. 2, p. 247-265, jul. 2011.

GODINO, J. D.; RIVAS, M.; CASTRO, W.; KONIC, P. Desarrollo de competencias para el análisis didáctico del profesor de matemáticas. REVEMAT, Florianópolis, v. 7, n. 2, p. 1-21, 2012.

GODINO, J. D.; BATANERO, C.; FONT, V.; GIACOMONE, B. Articulando conocimientos y competencias del profesor de matemáticas: el modelo CCDM. In: FERNÁNDEZ, C. et al. (Ed.). Investigación en Educación Matemática XX. Málaga: Ed. SEIEM, 2016. p. 288-297.

HILL H. C.; BALL D. L.; SCHILLING S. G. Unpacking pedagogical content knowledge: conceptualizing and measuring teachers' topic-specific knowledge of students. Journal for Research in Mathematics Education, Reston, v. 39, n. 4, p. 372-400, jul. 2008. 
JAWORSKI, B.; WOOD, T. (Ed.). The International Handbook of Mathematics Teacher Education. 1. ed. Rotterdam: Sense Publisher, 2008. 330 p.

KILPATRICK, J.; SWAFFORD, J.; FINDELL, B. (Ed.). Adding it up: helping children learn mathematics. 1. ed. Washington: National Academy Press, 2001. 480 p.

LO, J. J.; LEATHAM, K. R.; ZOEST, L. R. VAN. (Ed.). Research Trends in Mathematics Teacher Education. 1. ed. Berlín: Springer, 2014. 319 p.

MINISTERIO DE EDUCACIÓN, CULTURA Y DEPORTE. Orden ECD/65/2015, de 21 de enero, por la que se describen las relaciones entre las competencias, los contenidos, los criterios de evaluación de la educación primaria, la educación secundaria y el bachillerato. Boletín oficial del estado, Gobierno de España, n. 25, p. 6986-7003, enero. 2015.

NATIONAL GOVERNORS ASSOCIATION CENTER FOR BEST PRACTICES AND THE COUNCIL OF CHIEF STATE SCHOOL OFFICERS. Common Core State Standards for Mathematics. 2011. Disponible en: <http://www.corestandards.org/assets/CCSSI_Math\%20Stan dards.pdf $>$. Acceso en: 9, apr. 2016.

PINO-FAN, L.; GODINO, J. D. Perspectiva ampliada del conocimiento didáctico-matemático del profesor. Paradigma, Maracay, v. 36, n. 1, p. 87-109, mar. 2015.

PONTE, J. P.; CHAPMAN, O. Mathematics teachers' knowledge and practices. In: GUTIERREZ, A.; BOERO, P. (Ed.). Handbook of Research on the Psychology of Mathematics Education: past, present and future. Rotterdam: Ed. Sense Publisher, 2006. p. 461-494.

POSADAS, P.; GODINO, J. D. Reflexión sobre la práctica docente como estrategia formativa para desarrollar el conocimiento didáctico-matemático. Departamento de Didáctica de la matemática, Universidad de Granada, 2014. 21 p. Disponible en: 〈http://www.ugr.es/ jgodino/fprofeso res/Posadas_reflexion.pdf>. Acceso en: 9, apr. 2016.

ROWLAND, T.; HUCKSTEP, P.; THWAITES, A. Elementary teachers' mathematics subject knowledge: the knowledge quartet and the case of Naomi. Journal of Mathematics Teacher Education, Dordrecht, v. 8, n. 3, p. 255-281, jun. 2005.

RUBIO GOYCOCHEA, N. V. Competencia del profesorado en el Análisis Didáctico de Prácticas, Objetos y Procesos Matemáticos. 2012. 446 f. Tesis (Doctorado en Didàctica de les ciències experimentals i de la matemàtica) - Facultat de Formació del Professorat, Universitat de Barcelona, Barcelona, 2012.

SCHEINER, T. Lessons we have (not) learned from past and current conceptualizations of mathematics teachers' knowledge. In: KRAINER, K.; VONDROVÁ, N. (Ed.). CONGRESS OF EUROPEAN RESEARCH IN MATHEMATICS EDUCATION, 9. 2015, Prague. Proceedings... Prague: ERME 9, 2015. p. 3248-3253.

SCHOENFELD, A.; KILPATRICK, J. Towards a theory of proficiency in teaching mathematics. In: TIROSH, D.; WOOD, T. L. (Ed.). Tools and Processes in Mathematics Teacher Education. Rotterdam: Ed. Sense Publisher, 2008. p. 321-354.

SOWDER, J. T. The mathematical education and development of teachers. In: LESTER, F. K. (Ed.). Second Handbook of Research on Mathematics Teaching and Learning. Charlotte: Ed. NCTM and IAP, 2007. p. 157-224. 
WILHELMI, M. R.; GODINO, J. D.; LACASTA, E. Configuraciones epistémicas asociadas a la noción de igualdad de números reales. Recherches en Didactique des Mathematiques, Grenoble, v. 27, n. 1, p. 77-120, 2007.

Submetido em Abril de 2016. Aprovado em Dezembro de 2016. 